Journal of Engineering and Applied Sciences 15 (7): 1598-1603, 2020

ISSN: 1816-949X

(C) Medwell Journals, 2020

\title{
A Review of Batik Pattern Generations Methods
}

\author{
Dyah Rizky Hujairha Laitupa, Tito Waluyo Purboyo and Purba Daru Kusuma \\ Department of Computer Engineering, Faculty of Electrical Engineering, \\ Telkom University, Bandung, Indonesia
}

\begin{abstract}
The art of coloring the cloth with the coloring blocking technique using the night is one of the ancient art forms. And batik is one of the art of coloring cloth that has developed in Indonesia, since, Majapahit kingdom. The process of making batik traditionally takes a long time. From the length of the process of making batik there are stages that usually takes quite a long time called scratching is to draw a motive either on paper or directly on the cloth. This is because at the stage of scraping batik should think of ideas after getting new ideas batik will apply it. The process of getting this idea make the process longer in making batik. With the development of technology and science today, it can shorten making the pattern by using an algorithm or computation method.
\end{abstract}

Key words: Batik pattern, scratching, computation method, cloth, blocking technique, scratching

\section{INTRODUCTION}

Batik is an art form of staining cloth with a barrier technique using wax where the technique is an ancient art form. The discovery of a mummy wrapping cloth coated wax to form a pattern in Egypt shows that this technique has been known, since, the 4th century BC. In Asia, similar techniques of batik were also applied in China during the T'ang dynasty (618-907) and in India and Japan during the Nara period (645-794). In Africa, techniques such as batik are known by the Yoruba tribe in Nigeria as well as the Soninke and Wolof tribes in Senegal.

Batik pattern has uniqueness and beauty that become one of the character forming of Indonesia that distinguish us with other nation, so that can become identity and national identity. Batik is a downward art that has become a tradition in Indonesia. So that, the pattern of batik that can be recognized from certain areas and also to date can show the status of the wearer because some traditional pattern are only used by the family palace.

With the development of digital technology and science today batik pattern can be made with computational methods. And make the process scratching faster then the traditional way. Pattern batik will made by sequence of program code that implements the computational method to create also develop a varied batik pattern.

Computation method is one useful tool to modify traditional pattern. Some studies in computer science have been done to explore batik pattern (Kusuma, 2017). Rangkuti has studied batik pattern classification based on the content of the pattern (Rangkuti et al., 2014) and one of them used wavelet transform and fuzzy neural network
(Rangkuti, 2014). The other studies have made modification by extracting geometric features of the original patterns and then made some modifications in geometric features position (Minarno and Suciati, 2014) or in pattern complexity (Kusuma, 2017).

Kusuma has made modification in traditional regular pattern based on cellular automata model (Kusuma, 2017). One of them implemented pedestrian dynamic method (Kusuma, 2016). These studies explored regular pattern because of its simplicity, so, it can be easier to be made by computer program. The modifications have been done by adding some uncertainties to enrich the regular pattern. One of them implemented fully stochastic method (Kusuma, 2016) and the other implemented combination between stochastic and deterministic method (Kusuma, 2016). The new model is proposed to improve the occupied cell ratio problem. This model is developed by combining the modified interaction force and random walk (Kusuma, 2017). Interaction force method acts as collision avoidance part. In its origin, interaction force is a part of social forces model that is used to avoid collision between persons (Helbing and Molnar, 1995).

Develop fibrous root pattern in batik pattern generation by combining L-system and random walk. This research proposes realistic and simple root growth model. Then, this model is implemented to generate batik pattern. This model must be realistic, so, people still recognize that the pattern is root pattern. This model must be simple, so, the computation still light because this basic root pattern will be added into batik pattern generation application. In this research, the type of the root that is modeled is fibrous root (Kusuma, 2017).

All of them are built based on cellular automata model, fix iteration and multi agent system. The problem

Corresponding Author: Dyah Rizky Hujairha Laitupa, Department of Computer Engineering, Faculty of Electrical Engineering, Telkom University, Bandung, Indonesia 
in the previous researches is by using the small number of agents, the occupied cell ratio was still below $50 \%$. It is because the agents cannot move anymore before the iteration stops (Kusuma, 2017). So, increasing the number of occupied cells ratio to above $50 \%$ with same number of agents by extending travelling distance is still challenging.

Find out the potential of using mathematical and renewal methods in finishing batik patterns in new ways. We use algorithms or computational methods, expected to increase the creativity of artists to produce a variety of batik patterns. Here are the main points of focus in this study. First, features the main features in the pattern of batik making, so, it can be done gelatin got a new pattern. Secondly, merging between modern and kotemporen patterns. In the system is carried out search space design and development becomes larger. The system is then reproduced in detail and which has been previously adopted in the system. The results of the experiment resulted in the system can create and develop batik patterns into new patterns that are more effective and have greater potential (Li et al., 2009).

\section{MATERIALS AND METHODS}

Fractal dimension: There are two important things that can be defined batik is the process and pattern. When viewed from the process batik is a resist process for making designs on fabric. The artist uses wax to prevent dye from penetrating the cloth, leaving blank areas in the dyed fabric. The process, wax resist then dye can be repeated over and over to create complex multicolored designs. When viewed from the pattern, batik pattern consists of main patterns and ornaments of the main pattern filler. And usually the pattern of batik was repeated. And commonly used as batik patterns are plants, flowers, animals, nature and geometry. Batik is a textile art that has long developed in Indonesia in batik there is a functional value. Each pattern has a meaning and has different values. And in each region have different patterns and usually certain batik patterns are used for different rituals or events.

On the side of beauty can't judge a batik pattern is good or bad because it is subjective but in the process to understand the rules of manufacture of batik. The function of ornaments on the pattern of batik is not just to fill the space or complement the main pattern in batik but has its own value in the art of batik. In the pattern of batik must understand the meaning of the filler own batik patterns, not just make the lines and dots only. Through research found in batik pattern there is fractal.

Fractal is an abstract object that is used to describe and simulate natural objects and has several characteristics and differs from geometry. Self-similarity is the characteristic of artificial fractals. The invariant

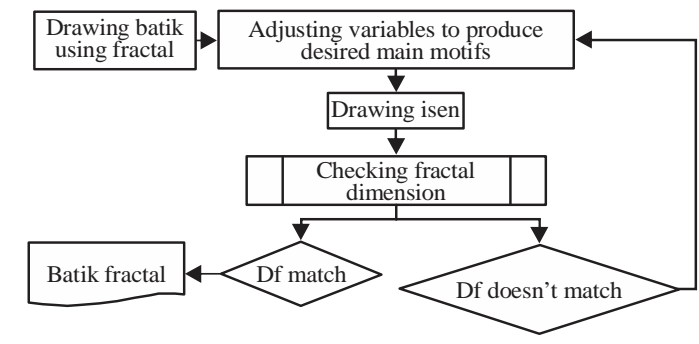

Fig. 1: Algorithm of making batik pattern with fractal

scale is the exact shape of the object at each magnification there is a smaller part of the object similar to the whole. Self-affinity is a characteristic of fractals whose pieces are scaled by different numbers in the $\mathrm{x}$ and $\mathrm{y}$ directions. This means that in order to appreciate the resemblance of these fractal objects they must be reset using anisotropic affine transformation.

In the process of making fractal batik is the manufacture of ornaments that fill the main pattern. The use of fractals can be implemented in several areas such as natural phenomena, fingerprint, health, seismology. Fractal can be the right method in making batik on batik there fractal. In Fig. 1 the algorithm is as follow:

In Fig. 2 and 3 is the process of making batik pattern using fractal, first step we made the main pattern and then the main pattern are duplicated and processed in such a way from the color, the slope or the size of the pattern. After we arranged in such a way the pattern will be added patternisen to be an additional ornament on the batik pattern.

Lindenmayer system: L-system is a formal rule arranged as grammatical in the form of axioma where symbols used represent growth of plants, parallel changes in symbols and stimulants occur at each stage (Suhartono et al., 2011). The main concept of L-system itself is rewriting. With the rewrite technique we will get a complex object by rewriting based on the defined rule (rewriting rule). For example, the application of rewriting L-system snowflake curve in 1905 by von Koch (Suhartono et al., 2011). The process involved involves the generator on the initiator, then applying the generator to the final output and so on (Fig. 4 and 5).

Symbol-symbol are used as representations of the growth of plant parts in parallel and simultaneous ones is turtle graph which can move in various directions, towards the front, towards the right or towards the left can be exemplified there are 3 symbols namely $\mathrm{F},+$ and -, diamana for the symbol $\mathrm{F}$ states a long part of the plant moving in various directions, clock rotation is expressed as + symbol and reverse direction as symbol (Suhartono et al., 2011).

There are many techniques in modeling plant growth. One of them is L-system that was introduced by 


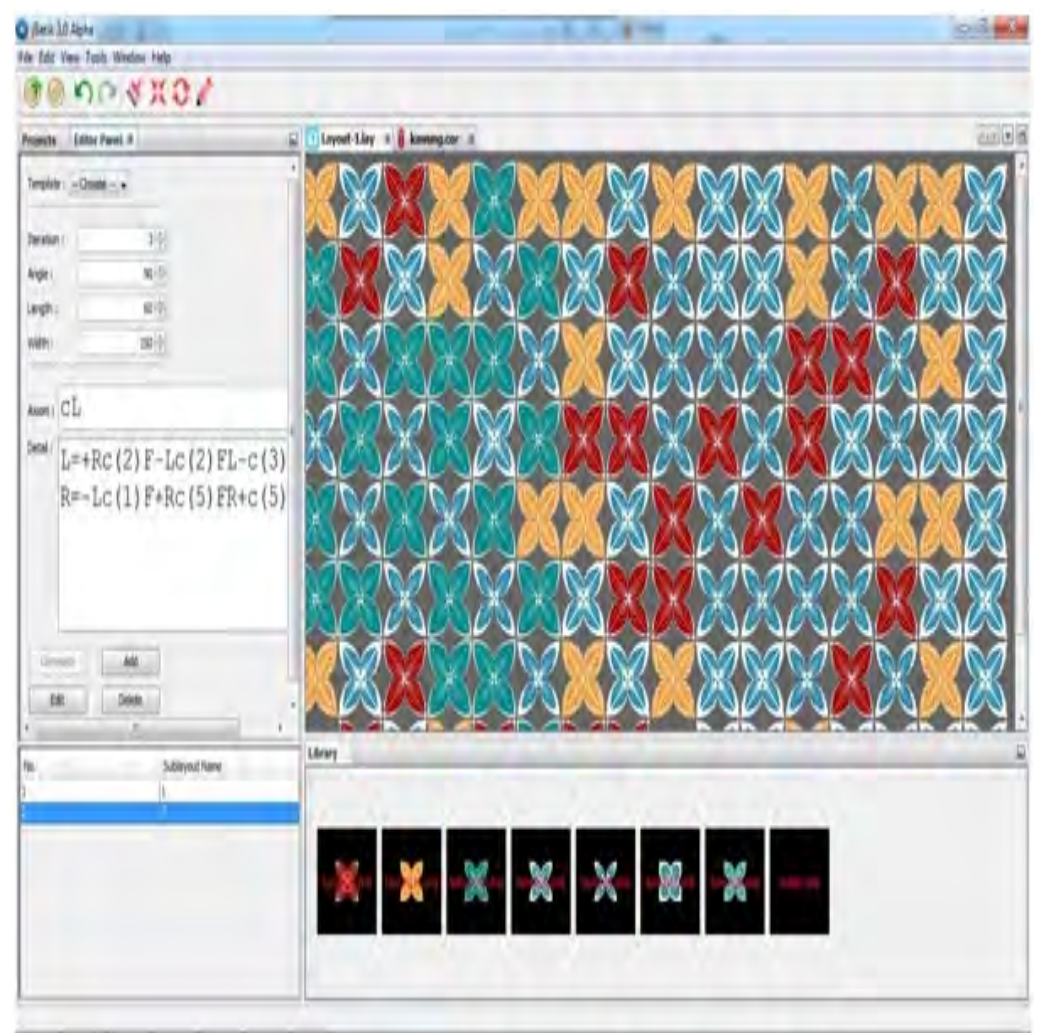

Fig. 2: Design batik pattern fractal 1

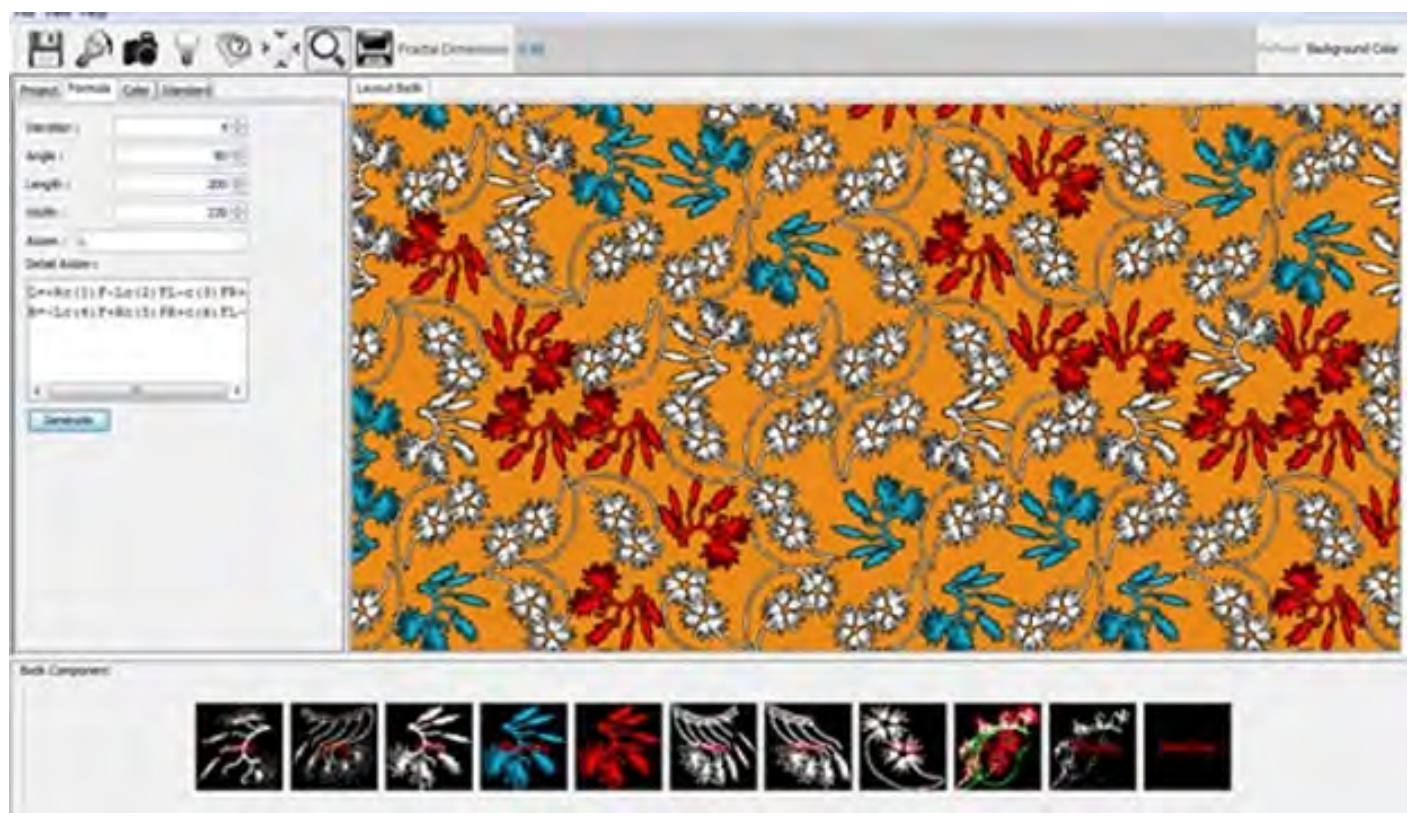

Fig. 3: Design batik pattern fractal 2

Lindenmayer. This method varies from simple to complex form (Prusinkiewicz et al., 1990). This method has been used to model root (Suhartono et al., 2013), branch, leaf and flower. This method also has been improved and combined with other method (Castellanos et al., 2014). L-system was also used to develop plant like 

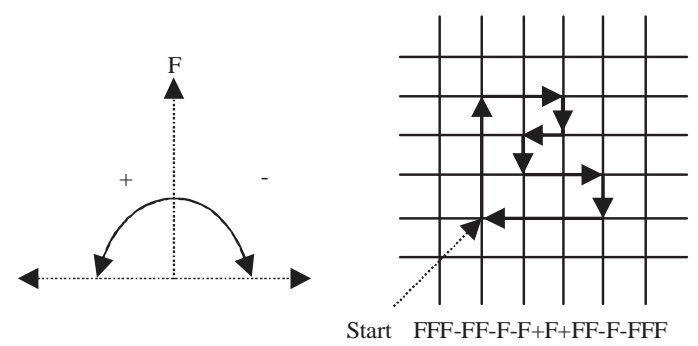

Fig. 4: Graph of direction

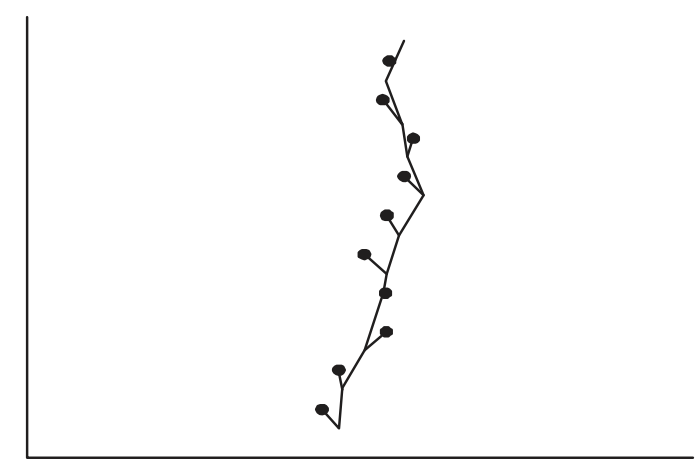

Fig. 5: Visualization example of simple L-system (Kusuma, 2017)

begin
node $[0] \leftarrow$ branch
status $[0] \leftarrow$ life
for $\mathrm{i}=1$ to $\mathrm{n}$
$\mathrm{m} \leftarrow$ length (node) -1
for $\mathrm{j}=0$ to $\mathrm{m}$
if node $[\mathrm{j}]=$ branch and
status $[\mathrm{j}]=$ life then
$\quad$ node $[\mathrm{m}] \leftarrow$ flower
$\quad$ node $[\mathrm{m}+1] \leftarrow$ branch
status $[\mathrm{j}] \leftarrow$ die
end
end
end
end

Fig. 6: Example of simple L-system alogoritham (Kusuma, 2017)

model. The other method is random walk. The other method is random walk that was used with the assumption that plant growth is stochastic and the plant behavior cannot be predicted exactly even many parameters have been used (Hanafin et al., 2011). Other method used complex biological mechanism such as elongation, mortality and gravity (Meng et al., 2011).

In Fig. 6 is based on this simple method, L-system then was used, modified and combined with other method to model more specific plant growth. Suhartono combined L-system and Fuzzy Mamdani to model Zinnia elegans growth (Suhartono et al., 2011, 2013). Castellanos used L-system to model plant death process simulation (Castellanos et al., 2014). Used L-system to model to model wheat rooting (Meng et al., 2011). Hamon used L-system to model 3D virtual plant simulation (Kusuma, 2017). In Fig. 5 is visualization from example of simple algorithm L-system.

\section{RESULTS AND DISCUSSION}

Random walk: Mathematical object known as a stochastic process that starts at a certain point and moves to a randomly selected neighbor from which the same process will be executed. The direction of movement or displacement will be determined by the probability value. The basic example of a random path is a random walk on an integer line, $\mathbb{Z}$ which starts at 0 and on every move move +1 or -1 with the same probability. The term random walk was first introduced by Karl Pearson in 1905 (Kohls, 2016). They are used to model different phenomena in mathematics and physics that are widely applied in areas such as economics, ecology, biology, computer science and others.

There are many traditional decorative patterns in Indonesia. Some of them are regular pattern. The advantage of regular one is its simplicity and easiness to be built by iterative algorithm. The disadvantage is it is monotonous. So, stochastic method can be implemented into regular pattern to add uncertainty. In this study, the uncertainty was added by implementing combination of deterministic and stochastic approach into the regular pattern. The deterministic part is represented by modified interaction forces method. The stochastic part is represented by random walk method. This model also implemented multi agent approach by using two types of agent: starting point determinant and walker. In this study, walker still walks until it cannot walk anymore. There are two parameters that are tested, the traveled nodes and the occupied cells ratio. In this research by using interaction force as movement model, the occupied cell ratio is above $50 \%$ (Kusuma, 2017).

Random walk acts as uncertainty part. Random walk has been adopted in many researches in image processing to make uncertainty (Kusuma, 2017). Random walk has been adopted in many plant growth pattern generation researches (Kusuma, 2017). Random walk is used in climbing plants competing for space (Kusuma, 2017), root growth and maneuver and many more. These researches used directed random walk because the pattern is not full random because of some factors such as gravity or other plants (Kusuma, 2017). 
Table 1: Review and discussion

\begin{tabular}{|c|c|c|}
\hline Methods & Author's name & Discussion \\
\hline Fractal dimension & $\begin{array}{l}\text { Lukman. M, } \\
\text { Hariadi. Y, } \\
\text { Destiarmand.A.H }\end{array}$ & $\begin{array}{l}\text { Each batik pattern has a different fractal dimension. But the numbers are not too large } \\
\text { and many, batik patterns are usually available on fractals that have dimensions between } \\
1 \text { and } 2 \text {. The value is obtained from testing on every batik pattern that comes from every } \\
\text { region in Indonesia using ANOVA test. ANOVA from Variable Analysis. In this test } \\
\text { grouping batik patterns according to their fractal dimensions. Using the transformation } \\
\text { dimension using fourier transforms which can be used to calculate the dimensions of } \\
\text { two-dimensional images. Of these tests usually batik is available in dimensions of } 1.5 \text {. } \\
\text { The applications are built using the PHP programming language and allow you to be } \\
\text { able to use batik patterns using conventional methods }\end{array}$ \\
\hline Lindenmayer system & Kusuma $(2016,2017)$ & $\begin{array}{l}\text { The first step is generating object model as a set of nodes. The second step is placing } \\
\text { batik objects at the selected nodes. In the first step, the root model is generated by the } \\
\text { combination of simplified L-system. The output of the first step is a set of nodes with } \\
\text { their coordinate that describes the object form and the status of the node because each } \\
\text { status of nodes will be presented with certain batik object. In the second step, the set } \\
\text { of nodes will be filled with specific nodes, lines and or curves that form specific batik } \\
\text { objects. The output of the second step is the batik image that visualizes object }\end{array}$ \\
\hline Random walk & Kohls (2016) & $\begin{array}{l}\text { The action that is chosen by the agent is probabilistic. Ratio is the ratio that determines } \\
\text { the branch will split }\end{array}$ \\
\hline
\end{tabular}

\section{CONCLUSION}

Generating batik pattern computationally is still challenging. There are many research potentials in batik pattern generation based on natural phenomena beside living object. Rain, wind, cloud, stone relief can also be used as inspiration. The combination between natural phenomenon and living objects is also challenging. The key point is still creating object as real as possible and in the other hand, reducing calculation is also necessary, so that, the computation can be reduced as much as possible.

When using full random walk or the combination between random walk and interaction force, the occupied cell ratio is below $50 \%$. It is also become the other research finding in this study. By removing the desired movement force which is the destination point and the movement become fully deterministic, the occupied ratio is high. It means that the existence of the stochastic part reduces the occupied ratio.

There are many research potentials in traditional art pattern generation. This pattern generation may adopt research in natural science to create natural based art pattern such as: plant growth, wave or river flow. Creating pattern generation model based on animal print is also challenging. The key point is combining computation technique with natural science to create new art pattern or to renew traditional pattern.

\section{REFERENCES}

Castellanos, E., F. Ramos and M. Ramos, 2014. Semantic death in plant's simulation using Lindenmayer systems. Proceedings of the 2014 10th International Conference on Natural Computation (ICNC), August 19-21, 2014, IEEE, Xiamen, China, ISBN:978-1-4799-5151-2, pp: 360-365.
Hanafin, S., S. Datta and B. Rolfe, 2011. Tree facades: Generative modelling with an axial branch rewriting system. Proceedings of the CAADRIA 16th International Conference on Computer-Aided Architectural Design Research in Asia Circuit Bending, Breaking and Mending, April 27-30, 2011, Association for Computer-Aided Architectural Design in Asia, Asia, Hong Kong, China, ISBN:9789881902627, pp: 175-184.

Helbing, D. and P. Molnar, 1995. Social force model for pedestrian dynamics. Phys. Rev. E., 51: 4282-4286.

Kohls, M., 2016. Expected coverage of random walk mobility algorithm. Master Thesis, Department of Electrical Engineering and Information Technology, Technical University of Dortmund, Dortmund, Germany.

Kusuma, P.D., 2016. Implementation of pedestrian dynamic in cellular automata based pattern generation. Intl. J. Adv. Comput. Sci. Appl., 7: 65-70.

Kusuma, P.D., 2017. Fibrous root model in batik pattern generation. J. Theor. Appl. Inf. Technol., 95: 3260-3269.

Li, Y., C.J. Hu and X. Yao, 2009. Innovative batik design with an interactive evolutionary art system. J. Comput. Sci. Technol., 24: 1-9.

Meng, J., X.Y. Guo, S.L. Lu, B.X. Xiao and W.L. Wen, 2011. Modeling rooting in wheat using lindenmayer system. Proceedings of the 2011 International Conference on Intelligent Computation Technology and Automation (ICICTA) Vol. 2, March 28-29, 2011, IEEE, Shenzhen, Guangdong, China, ISBN:978-1-61284-289-9, pp: 121-124.

Minarno, A.E. and N. Suciati, 2014. Batik image retrieval based on color difference histogram and gray level Co-occurrence matrix. Telkomnika Telecommun. Comput. Electron. Control, 12: 597-604. 
Prusinkiewicz, P., L. Aristid and H. James,
1990. The Algorithmic Beauty of Plants. Springer, Berlin, Germany, ISBN:9783540972976, Pages: 228.

Rangkuti, A.H., 2014. Content based batik image classification using wavelet transform and fuzzy neural network. J. Comput. Sci., 10: 604-613.

Rangkuti, H., A. Harjoko and A.E. Putro, 2014. Content based batik image retrieval. J. Comput. Sci., 10: 925-934.
Suhartono, M. Hariadi and M.H. Purnomo, 2011. Integration of Artificial neural network into genetic L-system programming based plant modeling environment with mathematica. Int. J. Acad. Res., Vol. 3.

Suhartono, M.H., M.H. Purnomo, Z. Baiting, X. JIA and H. Niu et al., 2013. Plant growth modeling of Zinnia elegans JACQ using fuzzy Mamdani and L-system approach with Mathematica. J. Theor. Appl. Inf. Technol., 50: 1-6. 